\title{
The antitumor efficiency of combined electrochemotherapy and single dose irradiation on a breast cancer tumor model
}

\author{
Elham Raeisi ${ }^{1,2}$, Seyed Mahmoud Reza Aghamiri³, Azin Bandi ${ }^{3}$, Negar Rahmatpour ${ }^{3}$, \\ Seyed Mohammad Firoozabadi ${ }^{1}$, Sedigheh Amini Kafi-Abad ${ }^{4}$, Lluis M Mir ${ }^{5,6,7}$ \\ ${ }_{1}^{1}$ Department of Medical Physics, Faculty of Medical Science, Tarbiat Modares University, Tehran, Iran \\ 2 Faculty of Science, University of Geneve, Geneve, Switzerland \\ ${ }^{3}$ Division of Radiation Medicine, Shahid Beheshti University, Tehran, Iran \\ ${ }^{4}$ Department of Immunohematology, Research Center of Iranian Blood Transfusion Organization (IBTO), Tehran, Iran \\ ${ }^{5}$ CNRS, Orsay, Laboratoire de Vectorologie et Therapeutiques Anticancereuses, UMR 8203, 91405 Orsay cedex, France \\ ${ }^{6}$ Universite Paris-Sud, Laboratoire de Vectorologie et Therapeutiques Anticancereuses, UMR 8203, 91405 Orsay cedex, France \\ ${ }^{7}$ Institute Gustave Roussy, Laboratoire de Vectorologie et Therapeutiques Anticancereuses, UMR 8203, 114 rue E. Vaillant, \\ 94805 Villejuif cedex, France
}

Radiol Oncol 2012; 46(3): 226-232.

Received 2 December 2011

Accepted 5 May 2012

Correspondence to: Seyed Mahmoud Reza Aghamiri; Division of Radiation Medicine, Shahid Beheshti University, Evin Street, Po. Box: 1983963113, Tehran, Iran. Phone: +982122431819; Fax +982122431780; E-mail: SMR-Aghamiri@sbu.ac.ir; Lluis M Mir; CNRS UMR 8203, Institut Gustave Roussy, 114 rue E. Vaillant, 94805 Villejuif cedex, France. Phone: +33142114792; Fax +33142115245; E-mail: luismir@igr.fr;

Disclosure: No potential conflicts of interest were disclosed.

Background. The aim of this study was to investigate the antitumor effectiveness of electrochemotherapy with cisplatin combined with suboptimal radiotherapy doses. Tumor radiosensitization was evaluated on large invasive ductal carcinoma tumors in Balb/C mice.

Materials and methods. Tumors of an average volume of $630 \mathrm{~mm}^{3}$ were treated with cisplatin, electric pulses, radiotherapy, electrochemotherapy, alone as well as in appropriate combinations. Tumors were irradiated with Cobalt-60 y-rays at doses $3 \mathrm{~Gy}$ and $5 \mathrm{~Gy}$ in combination with electrochemotherapy using cisplatin. Controls included each of the treatments alone as well as the combination of the radiotherapy with electric pulses alone or with cisplatin alone. Antitumor effectiveness was evaluated by tumor growth delay, tumor-doubling time, inhibition ratio and the objective response rates.

Results. As anticipated, electrochemotherapy was more effective than the treatment with cisplatin alone or the application of the electric pulses alone. When treatments were combined with tumor irradiation at either 3 or $5 \mathrm{~Gy}$, the combination with electrochemotherapy was more effective: at 5 Gy, 2 animals out of 8 were in complete remission 100 days later. In general the higher $5 \mathrm{~Gy}$ dose of $\mathrm{Y}$-radiation was more effective than the lower one of $3 \mathrm{~Gy}$.

Conclusions. The results of our study demonstrate that irradiation doses, 3 Gy or 5 Gy, increase the antitumor effectiveness of electrochemotherapy with cisplatin on invasive ductal carcinoma tumors. Good antitumor results were achieved in experimental tumors with a size comparable to clinical lesions, demonstrating that this three-modality combined treatment is useful for the treatment of large lesions even at sub-optimal radiotherapy doses.

Key words: cisplatin; irradiation; electroporation; invasive ductal carcinoma; multimodalities

\section{Introduction}

Cisplatin is an anti-cancer chemotherapeutic drug that is administered to treat a large number of cancers such as those of the ovary, head and neck, cer- vix and bladder, carcinomas as well as small-cell and non-small-cell lung cancers. ${ }^{1-5}$ However, resistance to cisplatin is a clinical problem for the treatment of some tumors. ${ }^{6}$ After an initial response to chemotherapy, tumors subsequently show the 
minimal responsiveness to cisplatin. ${ }^{1,2,7}$ Besides direct cytotoxic effects, cisplatin has also the potential to enhance radiation-induced cell killing. ${ }^{8-10}$ Cisplatin as a radiosensitizer increases the damage to the nuclear DNA of malignant cells, enhancing the anti-neoplastic efficacy of the radiotherapy. ${ }^{9,10}$ The combination of cisplatin and radiation is a common treatment modality with synergistic effects for cancers. ${ }^{4,5,8,9,11}$ Several in vitro and in vivo studies have shown higher response rates, prolonged mean survival, increased survival rates, longer local recurrence-free survival rates, and considerable organ preservation with the use of cisplatin in combination with external radiotherapy. ${ }^{8-11}$ Indeed, it has been proven that cisplatin enhances the cytotoxicity of radiation on cells in vitro, on experimental tumors in vivo and in solid tumors in the clinic. ${ }^{12-14}$

Many studies have been performed to potentiate cisplatin antitumor effectiveness. One way to increase the radiosensitizing effect of cisplatin can result from the increase of the cisplatin intracellular accumulation. Several studies have been conducted using different drug delivery systems to increase the amount of cisplatin in the tumor cells. ${ }^{8,14-18}$ Electropermeabilization is a physical method that uses short and intense electric pulses to facilitate drug delivery into the cells. ${ }^{19,20}$ Electrochemotherapy (ECT) combines the administration of specific chemotherapeutic drugs (bleomycin or cisplatin) with the application of electric pulses to the tumors to locally increase drug uptake and thus drug effectiveness. ${ }^{17,21}$ Electrochemotherapy with bleomycin and cisplatin was elaborated in vitro, in vivo and in clinical trials. ${ }^{15,16,21-25}$ Besides permeability, electroporation has other effects like a transient and reversible reduction of the blood flow. ${ }^{26}$ This reduction may contribute to the entrapment of the drug in the tumor, thereby providing more time for the drug to act. As this effect would reduce drug wash-out from the tumor, it could play a role in antitumor efficiency.

It has been demonstrated that the electroporation of tumors increases the radiosensitizing effect of cisplatin on small experimental tumors. ${ }^{27,28}$ The enhanced radiosensitizing effect of cisplatin is actually due to the increased electroporationmediated cisplatin delivery into the tumor cells. ${ }^{29}$ These initial studies used an already efficient dose of radiotherapy (RT) which was delivered to SA1 fibrosarcoma transplanted in mice, combined to electrochemotherapy using cisplatin as well as small tumors in mice. ${ }^{27}$
The aim of the present study was to evaluate the efficiency of electrochemotherapy with cisplatin combined with a single radiotherapy dose on an animal model tumor of breast adenocarcinoma to determine the potentialities of this combination. Two sub-optimal radiotherapy conditions, 3 Gy or $5 \mathrm{~Gy}$, unable to generate long term partial responses in this tumor model, were applied in these combinations. Moreover, it was decided to treat very large experimental tumors, of an average volume of $630 \mathrm{~mm}^{3}$, to assess the efficacy of the treatment alone or in combination in a preclinical model exploring the possibility of efficiently treating large lesions.

\section{Materials and methods}

\section{Mice and tumors}

Female Balb/C mice, purchased from the Pasteur Institute (Tehran, Iran) were maintained at $25^{\circ} \mathrm{C}$ with natural day/night cycle for 10 days for adaptation. Mice were 6-8 weeks old at the beginning of the experiments. A fragment $\left(4 \mathrm{~mm}^{3}\right)$ of a spontaneous mouse mammary tumor (an invasive ductal carcinoma) was transplanted subcutaneously into the flank of each animal. ${ }^{30,31}$ Two weeks after the implantation, when the tumors reached approximately $630 \mathrm{~mm}^{3}$ in volume $(12-15 \mathrm{~mm}$ in diameter), the mice were randomly divided into the experimental groups (8-10 animals for each control and each experimental group). The study was approved by the local Research and Medical Ethics Committee, in accordance with the Shahid Beheshti University of Medical Sciences Guidelines for the Care and Use of Laboratory Animals.

\section{Cisplatin}

Cisplatin (50 mg/ml, Ebewe Pharma, Austria) was diluted with $0.9 \% \mathrm{NaCl}$ the day of the treatment. Cisplatin at a dose of $8 \mathrm{mg} / \mathrm{kg}$ was injected intratumorally and the injected volume was adjusted to deliver $0.02 \mathrm{ml}$ of this cisplatin solution per gram of body weight.

\section{Electrochemotherapy}

Two flat stainless steel plates were mounted on a caliper to serve as $20 \times 20 \mathrm{~mm}$ parallel electrodes. A distance between electrodes was adjusted by rolling of the caliper depending on the tumor size. The voltage value was then set in an ECT-SBDC device (with a maximal output voltage of $1400 \mathrm{~V}$ ) to gen- 
TABLE 1. Tumor growth after treatment with three modalities; cisplatin, electric pulses and local $\mathrm{y}$-radiation or combination on invasive ductal carcinoma tumor model

\begin{tabular}{|c|c|c|c|c|c|}
\hline Experimental groups & $\mathrm{n}$ & $\begin{array}{c}\text { DT } \\
\text { days, Mean } \pm S E\end{array}$ & $\begin{array}{c}\text { GD } \\
\text { days, mean }\end{array}$ & Inhibition ratio (\%) & $\begin{array}{c}\text { CR } \\
\text { number }\end{array}$ \\
\hline Control & 8 & $4.6 \pm 0.5$ & & 0 & 0 \\
\hline CDDP & 8 & $10.6 \pm 1.21$ & 5.5 & 33 & 0 \\
\hline EP & 8 & $6.6 \pm 0.5$ & 2.0 & 32 & 0 \\
\hline ECT & 10 & $20.1 \pm 1.2$ & 15.5 & 61 & 0 \\
\hline IR (3 Gy) & 9 & $15.7 \pm 1.4$ & 11.1 & 63 & 0 \\
\hline$E P+I R$ (3 Gy) & 8 & $13.4 \pm 2.1$ & 8.8 & 51 & 0 \\
\hline$E C T+I R(3$ Gy) & 10 & $30.3 \pm 2.6$ & 25.7 & 76 & 0 \\
\hline IR (5 Gy) & 8 & $25.2 \pm 1.1$ & 20.6 & 70 & 0 \\
\hline CDDP + IR (5 Gy) & 8 & $25.6 \pm 1.2$ & 21.0 & 75 & 0 \\
\hline
\end{tabular}

$\mathrm{CDDP}=$ cisplatin; $\mathrm{EP}=$ electric pulses; $\mathrm{ECT}=$ electrochemotherapy; $\mathrm{IR}=$ irradiation; $\mathrm{n}=$ number of mice in the experimental group; $\mathrm{DT}=\mathrm{tumor}$ doubling time; $\mathrm{GD}=\mathrm{growth}$ delay; $\mathrm{PR}=$ partial response; $\mathrm{CR}=$ complete response

erate electric pulses with a voltage to distance ratio of $1000 \mathrm{~V} / \mathrm{cm}$. The electrodes and the ECT-SBDC device were designed in the electromagnetic laboratory of the Medical Physics department of Tarbiat Modares University, Tehran, Iran. Eight squarewave electric pulses (two trains of 4 pulses) of 1000 $\mathrm{V} / \mathrm{cm}$ amplitude, with a pulse duration of $100 \mu \mathrm{s}$ and repetition frequency $1 \mathrm{~Hz}$, were delivered by the two parallel electrodes which were placed on the skin at the opposite sides of the tumor. An optimal contact between the electrodes and the skin was assured by means of a conductive gel. In all the electrochemotherapy groups, mice were treated with the electric pulses $1 \mathrm{~min}$ after the cisplatin injection.

\section{Radiotherapy}

A cobalt-60 unit (Theraton 780, Canada) was used to locally deliver a single dose of $3 \mathrm{~Gy}$ or 5 Gy at a dose rate of $0.6 \mathrm{~Gy} / \mathrm{min}$ ( $3 \mathrm{~Gy}$ in $5 \mathrm{~min}$ ) or $0.71 \mathrm{~Gy} / \mathrm{min}$ (5 Gy in $7 \mathrm{~min}$ ). Irradiation was performed frontally. As the smallest field covered by the Cobalt beam was $4 \times 4 \mathrm{~cm}^{2}$, the mouse's body around the tumor was shielded using lead blocks so as to leave only the tumor exposed to the irradiation beam.

\section{Study protocol}

The experimental groups included: untreated tumors as controls, tumors treated with cisplatin, electric pulses or radiotherapy alone, tumors treated by electrochemotherapy (that is cisplatin injection followed by the local application of electroporative electric pulses) and tumors treated with the combination of cisplatin or electric pulses and radiotherapy. In the combination of cisplatin or electroporation with local radiotherapy, tumor-bearing mice were irradiated $30 \mathrm{~min}$ after the cisplatin injection or the electric pulses delivery, to provide the time to cisplatin to enter the cells.

\section{Treatment evaluation}

Tumor growth was monitored, every three days, by measuring two mutually orthogonal tumor diameters (e1 being the larger tumor diameter and e2 the largest diameter orthogonal to e1). Tumors were rather spherical and tumor volumes were calculated by the formula $\mathrm{V}=\pi / 6 \times \mathrm{e} 1 \times \mathrm{e} 2^{2}$. From the tumor growth curves, tumor-doubling time (DT) was determined for each individual tumor. Tumor growth delay (GD) was calculated by subtracting the mean tumor volume doubling time of the untreated tumors (control) from the mean tumor volume doubling time of each experimental group. Inhibition ratio, expressed in percent, was calculated at day 30 after the treatment by the formula [1(treated tumor average volume/untreated tumor average volume)] x100\%. Partial response (PR) was a decrease by more than $50 \%$ of the tumor volume. Complete response (CR) was the absence of tumor for more than 100 days. 
Normalized volumes at day $\mathrm{N}$ for each animal were calculated by dividing the tumor volume $\mathrm{Vn}$ at day $\mathrm{N}$ after the treatment by the average tumor volume $\mathrm{V} 0$ at day 0 that is at the day of the treatment $(\mathrm{Vn} / \mathrm{V} 0)$.

\section{Statistical analysis}

All data were tested for normality of distribution. The ANOVA test with repeated measures was used to evaluate statistical significance of differences between experimental and control groups at different times. A p-value $<0.05$ was considered significant in the statistical tests $\left({ }^{*} \mathrm{p}<0.05\right)$.

\section{Results}

As shown in Table 1 and Figure 1, cisplatin (at a dose of $8 \mathrm{mg} / \mathrm{kg}$ ) as a single treatment delayed the tumor growth up to 5.5 days with an inhibition ratio of $33 \%$. Tumors that were treated with electric pulses alone $(8$ pulses, $1000 \mathrm{~V} / \mathrm{cm}, 100 \mu$ s duration, at $1 \mathrm{~Hz}$ repetition frequency) displayed a tumor growth delay of only 2 days with an inhibition ratio of $32 \%$ (Table 1 ) and with no partial or complete responders with neither of both treatment modalities.

In the electrochemotherapy group, in which tumors were exposed to electric pulses $1 \mathrm{~min}$ after the cisplatin injection, a prolonged tumor growth delay (up to 15.5 days) was observed ( $<<0.05)$. The inhibition ratio reached $61 \%$ (Table 1 ). Thus, electrochemotherapy was more effective than either cisplatin alone or the application of electric pulses alone, with moreover $30 \%$ partial response for the first 9 days $(\mathrm{p}<0.05)$.

With local irradiation alone (Table 1 and Figures 1 and 2), the higher dose (5 Gy) resulted in a growth delay up to 20.6 days. Tumors irradiated with a single dose of 3 Gy resulted in a tumor growth delay of 11.1 days and an inhibition ratio of $63 \%$ (Table 1 ). Some partial responses $(25 \%)$ were observed within the 15 days that followed the administration of 5 Gy, but only $11 \%$ with 3 Gy.

When the administration of cisplatin was combined with local irradiation performed $30 \mathrm{~min}$ after the drug injection, the treatment of tumors resulted in tumor growth delays and inhibition ratios of 11.3 days and $61 \%$ respectively with a 3 Gy radiation and of 21.0 days and $75 \%$ with a 5 Gy irradiation (Table 1). These results demonstrate that a dose of 5 Gy was more effective. This is also borne out by the fact that $25 \%$ partial responses were observed

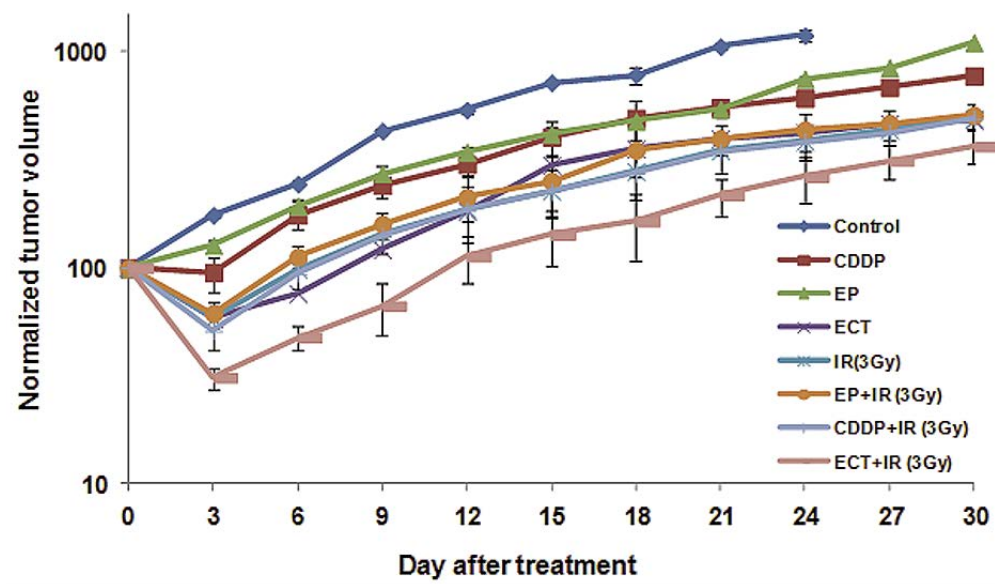

FIGURE 1. Tumor growth curves in untreated tumors or after treatment with CDDP (CDDP), electric pulses (EP) only, irradiation (IR) only, electrochemotherapy (ECT) and the combinations of CDDP or electric pulses and radiotherapy. Data are mean \pm SE of at least 8 animals for each of the experimental groups. Irradiation of tumor was $3 G y$.

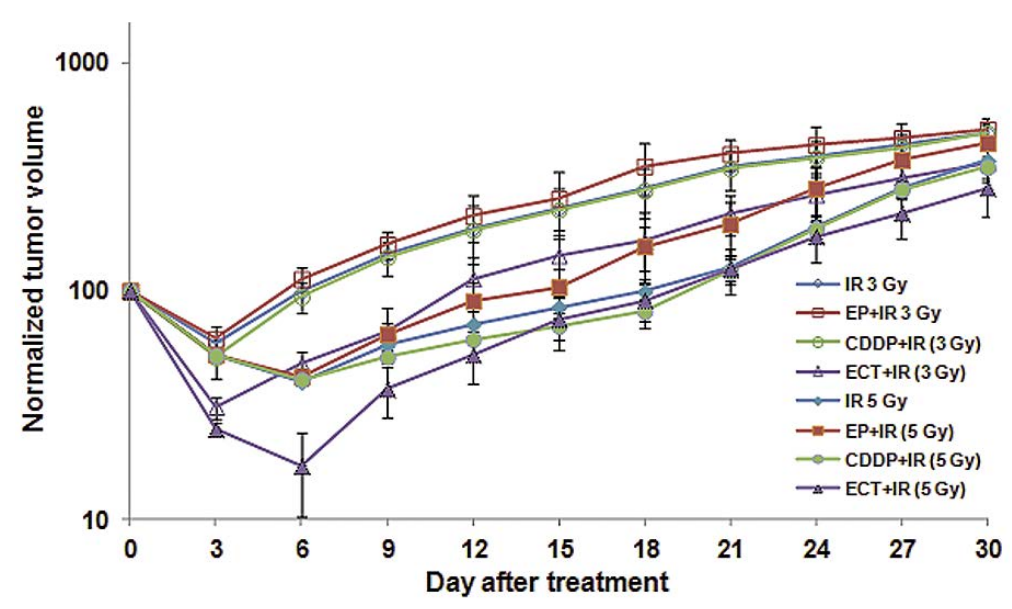

FIGURE 2. Tumor growth curves for invasive ductal carcinoma tumors after treatment with radiotherapy (IR) only, electrochemotherapy (ECT), combination of CDDP or electric pulses and radiotherapy at dose of $3 \mathrm{~Gy}$ in comparison to $5 \mathrm{~Gy}$. Data are mean \pm SE of at least 8 animals for each of the experimental groups.

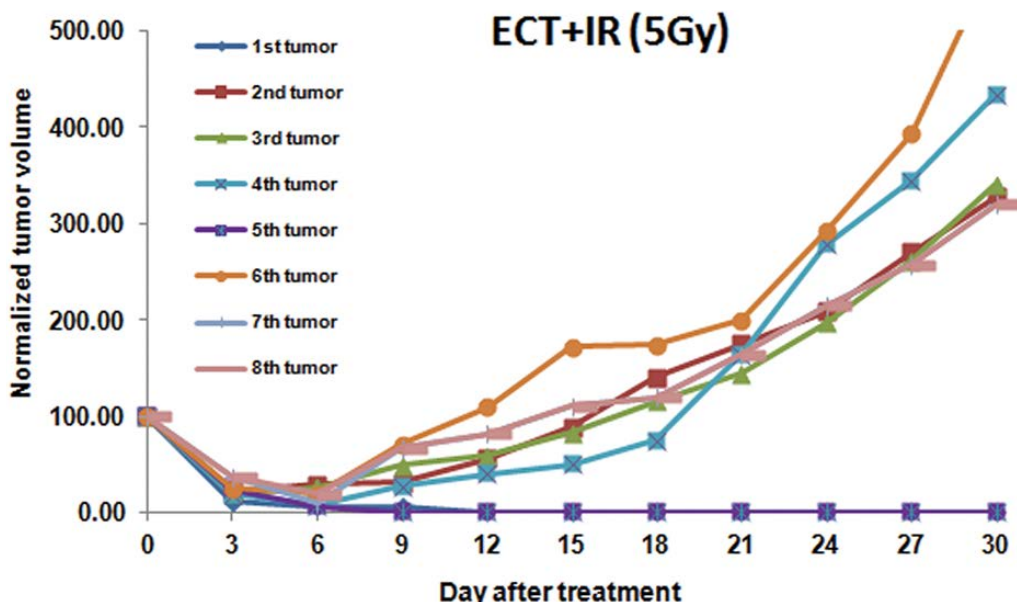

FIGURE 3. Individual tumor growth curves of tumors treated by the combination of electrochemotherapy (ECT) and radiotherapy at the dose of $5 \mathrm{~Gy}$. 
for up to 15 days with the 3 Gy irradiation, whereas only $11 \%$ partial responses were recorded up to 12 days following the combination of cisplatin and 3 Gy irradiation $(\mathrm{p}<0.05)$.

When electric pulses were combined with local irradiation delivered $30 \mathrm{~min}$ after the electric pulses application, an improved antitumor effect was not observed compared to irradiation alone. Tumor growth delays of 8.8 days and inhibition ratio of $51 \%$ after $3 \mathrm{~Gy}$ and of 17.8 days and 59\% after 5 Gy were scored (Table 1). With $5 \mathrm{~Gy}$, one partial response $(12.5 \%)$ was achieved for 15 days with no partial responders after $3 \mathrm{~Gy}$.

Irradiation of tumors pretreated with electrochemotherapy prolonged the tumor growth delay up to 25.7 days and 38.6 days at 3 Gy and 5 Gy respectively. The inhibition ratio reached $76 \%$ for the 3 Gy irradiation and $87 \%$ for $5 \mathrm{~Gy}$. Ten per cent of partial responses after 30 days were observed with electrochemotherapy followed by $3 \mathrm{~Gy}$, though without complete responders. In contrast, the electrochemotherapy with a 5 Gy irradiation resulted in a higher percentage of partial regressions (37\% of the tumors were still in partial response 30 days after the treatment). Moreover, $25 \%$ of the mice were still in complete remission 100 days after the treatment (Table 1 and Figure 3). Again, the combination with 5 Gy was significantly $(p<0.05)$ more efficient than the combination with $3 \mathrm{~Gy}$. Even in this group, no body weight loss or skin desquamation was reported. Only a hair loss was observed in all the groups that were irradiated, locally at the level of the irradiation site.

In summary, in all the cases, the combination with $\gamma$-radiation resulted in a larger antitumor efficacy than without the combination with $\gamma$-rays.

\section{Discussion}

In this study, the therapeutic effects of cisplatin, electroporation, $\gamma$-radiation and combination of these treatments were evaluated in a murine invasive ductal carcinoma tumor model. Our data confirm and extend previous findings ${ }^{16-18}$ showing that the delivery to tumors of electric pulses and cisplatin enhances the cisplatin-induced radiosensitization.

Electroporation combined with cisplatin has shown a significant effectiveness both in vitro and in vivo, as well as in clinical studies in the treatment of patients with cutaneous tumor nodules. ${ }^{15-18,24,25}$ Although the increased radiosensitizing effect of cisplatin using electroporation has been shown in
EAT carcinoma and LPB sarcoma cells ${ }^{27,28}$, data on radiosensitization for large tumors, of a size closer to clinical situations, have not yet been reported. In this study, this issue was addressed and using local $\gamma$-radiation with single doses of $3 \mathrm{~Gy}$ or $5 \mathrm{~Gy}$ of Cobalt-60 $\gamma$-rays in combination with electrochemotherapy with cisplatin on large invasive ductal carcinoma tumors as a model for the treatment of large breast tumors.

Our study confirms that electrochemotherapy was more effective than individual treatments using cisplatin or electric pulses alone which is consistent with previous reports. ${ }^{16-18,27,28}$ When electrochemotherapy with cisplatin was combined with local irradiation, the antitumor efficiency was doubled. This three-modality therapy was better than electrochemotherapy, cisplatin combined with local tumor irradiation and electric pulses combined with irradiation. In addition, some tumors were still in complete remission 100 days after the treatment at single dose of $5 \mathrm{~Gy}$. It is important to highlight that the irradiation doses used in the present study (a single 3 Gy or 5 Gy dose) are lower than the doses used in the previous experimental studies with cisplatin reported here above. ${ }^{27,28}$ Indeed, Sersa et al. used a dose of 15 Gy for electrochemotherapy with cisplatin combined with irradiation on the EAT tumor animal model. ${ }^{27}$ However, there are studies that demonstrated an increase of the tumor curability dose (TCD50) for both cisplatin (being 1.6) and bleomycin (1.9). . $^{32,33}$

This study concentrated on electrochemotherapy with cisplatin, as cisplatin is a radiosentizer, with the aim also to show that electrochemotherapy can radiosensitize the tumors. However, the initial goal was to determine whether electrochemotherapy efficacy could be increased by irradiation even in large tumors. Indeed, using about the same dose rate 2.2 Gy/min (10-20 Gy), Sersa et al. showed that efficacy of electrochemotherapy with bleomycin (a molecule considered a radiomimetic but not a radiosensitizer) was increased with irradiation, on SA-1 and CaNT tumor models. ${ }^{32}$

Finally it is important to report that our results are also in agreement with two clinical studies $^{34,35}$ reporting that electrochemotherapy in combination with irradiation is an effective and safe treatment for tumors of various origins in patients, thus on lesions of a size at least comparable to those treated in this study.

In conclusion the results of this study demonstrate that electrochemotherapy with cisplatin radiosensitized the tumors to a single low irradiation dose (3 Gy or 5 Gy of Cobalt- $60 \gamma$-rays) on invasive 
ductal carcinoma tumors. Moreover, this three-modality combined treatment was useful for the treatment of large lesions even at sub-optimal doses of irradiation. Complete response of very large tumors could be achieved after a single session with the combined multimodality treatment.

\section{Acknowledgements}

This work was supported by the Radiation Medicine Department, Shahid Beheshti University, Tehran, Iran. Authors would like to thank Novin Medical Radiation Institute (NMRI), Tehran, Iran especially Dr. Zahmatkesh and Dr. Akhlaghpour for providing us with Cobalt-60 therapy system. We also thank Professor Ahmadiani, Neuroscience Research Center, Shahid Beheshti University, Medical Sciences, Tehran, Iran. One of the authors (ER) would like to thanks the ABC Laboratory from the European Scientific Institute, 74160 Archamps, France, where the present manuscript was completed. Also, we would like to thank Prof. Miralbell from Dept. of Radiation Oncology, University Hospital, Geneve, Switzerland for help in editing the manuscript.

\section{References}

1. Gately DP, Howell SB. Cellular accumulation of the anticancer agent CDDP: a review. Br J Cancer 1993; 67: 1171-6.

2. Kelland $\mathrm{L}$. The resurgence of platinum-based cancer chemotherapy. Nat Rev Cancer 2007; 7: 573-84

3. Lekic M, Kovac V, Triller N, Knez L, Sadikov A, Cufer T. Outcome of small cell lung cancer (SCLC) patients with brain metastases in a routine clinical setting. Radiol Oncol 2012; 46(3): 226-232.; 46: 54-9.

4. Malecki K, Glinski B, Mucha-Malecka A, Rys J, Kruczak A, Roszkowski K, et al. Prognostic and predictive significance of p53, EGFr, Ki-67 in larynx preservation treatment. Rep Pract Oncol Radiother. 2010; 15: 87-92.

5. Kovac V, Smrdel U. Meta-analyses of clinical trials in patients with non-small cell lung cancer. Neoplasma 2004; 51: 334-40.

6. Erculj N, Kovac V, Hmeljak J, Dolzan V. The influence of platinum pathway polymorphisms on the outcome in patients with malignant mesothelioma. Ann Oncol 2012; 23: 961-7.

7. Kelland LR. Preclinical perspectives on platinum resistance. Drugs 2000; 59: 1-8.

8. Begg AC, Deurloo MJ, Kop W, Bartelink H. Improvement of combined modality therapy with CDDP and radiation using intratumoral drug administration in murine tumors. Radiother Oncol 1994; 31: 129-37.

9. Sharma VM, Wilson WR. Radiosensitization of advanced squamous cell carcinoma of the head and neck with CDDP during concomitant radiation therapy. Eur Arch Otorhinolaryngol 1999; 256: 462-5.

10. Bergs JWJ, Franken NAP, Cate RT, Bree CV, Haveman J. Effects of CDDP and $\gamma$-irradiation on cell survival, the induction of chromosomal aberrations and apoptosis in SW-1573 cells. Mutat Res 2006; 594: 148-54.

11. Ning S, Yu N, Brown DM, Kanekal S, Knox SJ. Radiosensitization by intratumoral administration of CDDP in a sustained-release drug delivery system. Radiother Oncol 1999; 50: 215-23.
12. Strojan P, Katarina K, Lojze S, Erika S, Igor F, Boris J, Aleksandar A, Marjan B, Branko Z. Concomitant chemoradiotherapy with Mitomycin $\mathrm{C}$ and Cisplatin in advanced unresectable carcinoma of the head and neck: Phase I-II Clinical Study. Int J Radiat Oncol Biol Phys 2008; 72: 365-72.

13. Carde P, Laval F. Effect of cis-dichlorodiammineplatinum (II) and X-rays on mammalian cell survival. Int J Radiat Oncol Biol Phys 1981; 7: 929-33.

14. Geldof AA, Slotman BJ. Radiosensitizing effect of CDDP in prostate cancer cell lines. Cancer Lett 1996; 101: 233-9.

15. Melvik JE, Pettersen EO, Gordon PB, Seglen PO. Increase in cis- dichlorodiammineplatinum (II) cytotoxicity upon reversible electro-permeabilization of the plasma membrane in cultured human NHIK 3025 cells. Eur J Cancer Clin Oncol 1986; 22: 1523-30.

16. Cemazar M, Miklavcic D, Vodovnik L, Jarm T, Rudolf Z, Stabuc B, et al. Improved therapeutic effect of electrochemotherapy with cisplatin by intratumoral drug administration and changing of electrode orientation for electromeatabilization on EAT tumor model in mice. Radiol Oncol 1995; 29: 121-7.

17. Sersa G, Cemazar M, Miklavcic D. Antitumor effectiveness of electrochemotherapy with cis-diamminedichloroplatinum (II) in mice. Cancer Res 1995; 55: 3450-5.

18. Sersa G, Cemazar M, Semrov D, Miklavcic D. Changing electrode orientation improves the efficacy of electrochemotherapy of solid tumors in mice. Bioelectrochem Bioenerg 1996; 39: 61-6.

19. Mir LM, Orlowski S. The basis of electrochemotherapy. In: Jaroszeski MJ, Heller R, Gilbert R, editors. Electrochemotherapy, Electrogentherapy, and Transdermal Drug Delivery. Electrically Mediated Delivery of Molecules to Cells. Totowa: Humana Press; 2000. p. 99-117.

20. Silve A, Mir LM. Clinical aspects of electroporation. In: Kee ST, Gehl J, Lee EW, editors. Cell electropermeabilization and cellular uptake of small molecules: the ECT concept. New York; Springer; 2011. p. 69-82.

21. Mir LM, Orlowski S, Belehradek J Jr, Paoletti C. Electrochemotherapy potentiation of antitumor effect of bleomycin by local electric pulses. Eur J Cancer 1991; 27: 68-72.

22. Marty M, Sersa G, Garbay JR, Gehl J, Collins CG, Snoj M, et al. Electrochemotherapy-an easy, highly effective and safe treatment of cutaneous and subcutaneous metastases: results of ESOPE (European Standard Operating Procedures of Electrochemotherapy) study. EJC Suppl 2006; 4: 3-13.

23. Sersa G, Stabuc B, Cemazar M, Jancar B, Miklavcic D, Rudolf Z Electrochemotherapy with CDDP: potentiation of local CDDP antitumor effectiveness by application of electric pulses in cancer patients. Eur J Cancer 1998; 34: 1213-8.

24. Cemazar M, Sersa G, Miklavcic D, Scancar J, Dolzan, Golouh R, et al Increased platinum accumulation in SA-1 tumor cells after in vivo electrochemotherapy with CDDP. Br J Cancer 1999; 79: 1386-91.

25. Sersa G, Stabuc B, Cemazar M, Miklavcic D, Rudolf Z. Electrochemotherapy with cisplatin: clinical experience in malignant melanoma patients. Clin Cancer Res 2000; 6: 863-7.

26. Jarm T, Cemazar M, Miklavcic D, Sersa G. Antivascular effects of electrochemotherapy: implications in treatment of bleeding metastases. Expert Rev Anticancer Ther 2010; 10: 729-46.

27. Sersa G, Kranjc S, Cemazar M. Improvement of combined modality therapy with CDDP and radiation using electroporation of tumors. Int J Radiat Oncol Biol Phys 2000; 46: 1037-41.

28. Kranjc S, Cemazar M, Grosel A, Scancar J, Sersa G. Electroporation of LPB sarcoma cells in vitro and tumors in vivo increases the radiosensitizing effect of CDDP. Anticancer Res 2003; 23: 275-82.

29. Kranjc S, Cemazar M, Grosel A, Pipan Z, Sersa G. Effect of electroporation on radiosensitization with cisplatin in two cell lines with different chemo- and radiosensitivity. Radio Oncol 2003; 37: 101-7.

30. Hassan ZM, Yaraee R, Chazanfari T, Safar Kejad AH, Nozari B Immunomodulatory affect of R10 fraction of garlic extract on natural killer activity. Int J Immunopharmaco 2003; 3: 1483-9.

31. Raeisi E, Firoozabadi SMP, Hajizadeh S, Rajabi H, Hassan Z M. The effect of high-frequency electric pulses on tumor blood flow in vivo. J Membrane Biol 2010; 236: 163-66. 
32. Kranjc S, Tevz G, Vidic S, Cemazar M, Sersa G. Radiosensitizing effect of electrochemotherapy in a fractionated radiation regimen in radiosensitive murine sarcoma and radioresistant adenocarcinoma tumor model. Radiat Res 2009; 172: 677-85.

33. Sersa G, Kranjc S, Cemazar M. Advanced electroporation techniques in biology and medicine. In: Pakhomov A, Miklavcic D, Marko MS, editors. Combined modality therapy: electrochemotherapy with tumor irradiation. CRC Press; 2010. p. 441-452.

34. Sersa G, Cemazar M, Rudolf Z, Fras AP. Adenocarcinoma skin metastases treated by electrochemotherapy with cisplatin combined with radiation. Radiol Oncol 1999; 33: 291-6.

35. Skarlatos I, Kyrgias G, Mosa E, Provatopoulou X, Spyrou M, Theodorou K, et al. Electrochemotherapy in cancer patients: first clinical trial in Greece. In Vivo 2011; 25: 265-74. 